Research Article

\title{
The Analysis of Using Spatial Smoothing for DOA Estimation of Coherent Signals in Sparse Arrays
}

\author{
Haiyun Xu (D), Weijia Cui ${ }^{D}$, Yuxi Du, Fengtong Mei ${ }^{D}$, and Bin Ba \\ National Digital System Engineering and Technological Research R \& D Center, Zhengzhou, Henan 450002, China \\ Correspondence should be addressed to Haiyun Xu; xuhaiyun1995@163.com
}

Received 14 May 2021; Revised 25 July 2021; Accepted 28 August 2021; Published 17 September 2021

Academic Editor: Francisco Falcone

Copyright (c) 2021 Haiyun Xu et al. This is an open access article distributed under the Creative Commons Attribution License, which permits unrestricted use, distribution, and reproduction in any medium, provided the original work is properly cited.

When there is coexistence of uncorrelated and coherent signals in sparse arrays, the conventional algorithms for direction-ofarrival (DOA) estimation using difference coarray fail. In order to solve the problems, this paper analyzes the feasibility of using spatial smoothing in sparse arrays. Firstly, we summarize the two types of sparse arrays, one consisting of identical sparse subarrays and the other consisting of several uniform linear subarrays. Then, we give the feasibility analysis and the processes of applying spatial smoothing. At last, we discuss the performance of the number of detectable coherent signals in different sparse arrays. Numerical experiments prove the conclusions proposed by the paper.

\section{Introduction}

Direction of arrival (DOA) is the one of the key parameters of the wireless positioning technique. It has been widely used in the fifth generation communication systems, military early warning, radar monitoring, sonar targets positioning, and so on $[1,2]$. Traditionally, the super-resolution DOA estimation methods, such as subspace methods, mainly use uniform nonsparse arrays like uniform linear arrays (ULAs). In recent years, many experts and scholars focus on nonuniform sparse arrays, which can provide larger array aperture with the same number of sensors. The typical sparse arrays are minimum redundancy arrays (MRAs) [3], coprime linear arrays (CLAs) [4], and nested linear arrays (NLAs) [5]. Through transforming the sparse array to a virtual ULA based on difference coarray, spatial smoothing multiple signal classification (SS-MUSIC) [6] and direct augmentation approach (DAA) [7] are proposed to solve DOAs. Moreover, compressed sensing can be directly applied to difference coarray to estimate DOAs [8-10].

Based on the DOA estimation methods mentioned above, many researchers proposed the improved array design methods. One design thought is based on the model of CLA. Coprime array with compressed interelement spacing (CACIS), coprime array with displaced subarrays (CADiS)
$[11,12]$, coprime array with multiperiod subarrays (CAMpS) [13], shifted coprime array (SCA) [14], generalized nested array (GNA) [15], and novel sparse arrays with two uniform arrays (NSA-U2) [16] were proposed, where they all consist of two ULA-subarrays and have larger array aperture than that of CLA. CACIS and CADiS show that setting one subarray with a smaller interelement spacing can have larger aperture of virtual ULA. CAMpS demonstrates that in order to expand the aperture of virtual ULA, only one subarray can have a compressed interelement spacing. SCA reveals that the displacement between two subarrays is the main factor to the aperture of virtual ULA. Although GNA has the same degree of freedom as NLA, it owns a sparser array structure. NSA-U2 presents the solution to have the maximum degree of freedom for sparse arrays with two uniform arrays. In order to further improve the array aperture, the sparse arrays with multiple ULA-subarrays are proposed. Super-nested arrays (SNAs) $[17,18]$, the augmented nested array (ANA) [19], and the maximum interelement spacing constraint (MISC) [20] divide the dense subarray of CLA into several sparse ULA-subarrays. Another design thought is combining several identical sparse arrays, such as nested MRA (NMRA) [21, 22], generalized nested subarray (GNSA) [23], and displaced multistage cascade subarrays (MSC-DiSA) [24]. The subarrays can be any sparse array and the 
displacement between the subarrays depends on sensors' location of another chosen sparse array. Although the aperture is smaller, the latter design method is less complex than that of the former method.

Unfortunately, the structure design of sparse arrays and DOA estimation algorithms are both based on the assumption that the impinging signals are uncorrelated to each other. But there exist coherent signals in real environment, such as in multipath channel. Many decoherence methods, such as spatial smoothing [25], forward/backward spatial smoothing (FBSS) [26], and Toeplitz reconstruction [27], are only applicable to the uniform structure arrays. Hence, the DOA estimation of coherent signals in sparse arrays has been a focus of interest. There have been some algorithms only for CLAs. Signal separation and Toeplitz reconstruction (SSTR) [28] and spatial smoothing using fourth-order cumulant (SS-FOC) [29] are all utilized in the uniform sparse subarrays and combined with common peak finding [30] to resolve the real values. Then, a method [31] combining spatial smoothing and matrix completion theory was proposed, which was applied to the data of physical sensors, but it also has strict restriction about array structure.

In order to solve coherent signals, we aim to give the process of using spatial smoothing in sparse arrays. We first summarize that the sparse array can be seen as two types: one is using several identical sparse arrays and the other consists of several ULA-subarrays. We then, respectively, apply the spatial smoothing and analyze the feasibility of decoherence methods. Next, we discuss the performance of the existing sparse arrays about the number of detectable coherent signals. At last, the simulation experiments are presented to prove the effectiveness of the proposed method.

The rest of this paper is organized as follows. Section 2 presents the model of received data including coherent and uncorrelated signals. Section 3 introduces the spatial smoothing process in sparse arrays. Section 4 gives the performance analysis and simulation experiments. Section 5 summarizes the paper. Throughout the paper, we make use of the notations shown in Table 1.

\section{Signal Model}

Suppose that there are $K$ far-field narrow-band signals impinging on a sparse array with $M$ sensors. Define the unit interelement spacing as $\lambda / 2$, where $\lambda$ is the wavelength of signals, and a integer set corresponding to the sensors location is given by $\mathbb{D}=\left\{0, \mathrm{~d}_{1}, \mathrm{~d}_{2}, \ldots, \mathrm{d}_{M-1}\right\}$ (generally assuming $\left.\mathrm{d}_{1}<\mathrm{d}_{2}<\cdots<\mathrm{d}_{M-1}\right)$. Assume that there are $P$ coherent signal groups, where the $p$ th group has $L_{p}$ signals. The coherent signal coming from $\theta_{p, \ell}$ is corresponding to the $\ell$ th multipath propagation of $S_{p}(t)$ with power $\sigma_{p}^{2}$ $(p=1, \ldots, P)$. The signals within each group are coherent to each other and uncorrelated to those in different groups. The total number of coherent signals is $K_{c}=\sum_{p=1}^{P} L_{p}$. In addition, the remaining signals, $S_{k}(t)$ coming from $\theta_{k}$ with the power $\sigma_{k}^{2}\left(k=K_{c}+1, \ldots, K\right)$, are uncorrelated to each other. The number of those signals is $K_{u}=K-K_{c}$. Thus, the received signals is
TABLE 1: Key notations used in this paper.

\begin{tabular}{lc}
\hline Notations & Explanation \\
\hline$(\bullet)^{T}$ & Transpose \\
$(\bullet)^{*}$ & Conjugate \\
$(\bullet)^{H}$ & Hermitian transpose \\
$\operatorname{gcd}(\bullet)$ & Greatest common divisor operation \\
$\operatorname{diag}[\bullet]$ & Diagonal matrix \\
$\mathbf{I}_{M}$ & Identity matrix with size $M \times M$ \\
$\operatorname{rank}(\bullet)$ & Rank of a matrix \\
$\lfloor\bullet\rfloor$ & Floor integer \\
\hline
\end{tabular}

$$
\begin{aligned}
\mathbf{X}(t) & =\sum_{p=1}^{P} \sum_{\ell=1}^{L_{p}} \mathbf{a}_{\mathbb{D}}\left(\theta_{p, \ell}\right) \beta_{p, \ell} S_{p}(t)+\sum_{k=K_{c}+1}^{K} \mathbf{a}_{\mathbb{D}}\left(\theta_{k}\right) S_{k}(t)+\mathbf{N}(t) \\
& =\mathbf{A}(\theta) \mathbf{S}(t)+\mathbf{N}(t)
\end{aligned}
$$

where the manifold matrix $\mathbf{A}(\theta)$ is denoted as

$$
\mathbf{A}(\theta)=\left[\mathbf{a}_{\mathbb{D}}\left(\theta_{1,1}\right), \ldots, \mathbf{a}_{\mathbb{D}}\left(\theta_{P, L_{P}}\right), \mathbf{a}_{\mathbb{D}}\left(\theta_{K_{c}+1}\right), \ldots, \mathbf{a}_{\mathbb{D}}\left(\theta_{K}\right)\right],
$$

and the steering vector $\mathbf{a}_{\mathbb{D}}\left(\theta_{k}\right)$ can be given by

$$
\mathbf{a}_{\mathbb{D}}\left(\theta_{k}\right)=\left[1, e^{-j \pi d_{1} \sin \theta_{k}}, \ldots, e^{-j \pi d_{M-1} \sin \theta_{k}}\right]^{T},
$$

and $\beta_{p, \ell}$ is the complex fading coefficient of the $\ell$ th coherent signal in the $p$ th group. The signal data vector is

$$
\mathbf{S}(t)=\left[\beta_{1,1} S_{1}(t), \ldots, \beta_{P, L_{P}} S_{P}(t), S_{K_{c}+1}(t), \ldots, S_{K}(t)\right]^{T},
$$

where $t=1, \ldots, J$, and $J$ is the number of snapshots. The noise vector is usually a Gaussian random variable with zero mean and variance $\sigma_{n}^{2}$.

From (1), the covariance matrix is denoted as

$$
\mathbf{R}_{X}=\frac{1}{J} \mathbf{X} \mathbf{X}^{H}=\mathbf{A R}_{S} \mathbf{A}^{H}+\sigma_{n}^{2} \mathbf{I}_{M},
$$

where $\mathbf{R}_{S}$ can be written as a block-diagonal matrix given by

$$
\begin{aligned}
\mathbf{R}_{S} & =\left[\begin{array}{cccccc}
\mathbf{R}_{1} & & & & & \\
& \ddots & & & & \\
& & \mathbf{R}_{P} & & & \\
& & \sigma_{K_{c}+1}^{2} & & \\
& & & \ddots & \\
& & & & \sigma_{K}^{2}
\end{array}\right], \\
\mathbf{R}_{p} & =\left[\begin{array}{ccccc}
\left|\beta_{p, 1}\right|^{2} \sigma_{p}^{2} & \beta_{p, 1} \beta_{p, 2}^{*} \sigma_{p}^{2} & \ldots & \beta_{p, 1} \beta_{p, L_{p}}^{*} \sigma_{p}^{2} \\
\beta_{p, 2} \beta_{p, 1}^{*} \sigma_{p}^{2} & \left|\beta_{p, 2}\right|^{2} \sigma_{p}^{2} & \ldots & \beta_{p, 2} \beta_{p, L_{p}}^{*} \sigma_{p}^{2} \\
\vdots & & \vdots & \ddots & \vdots \\
\beta_{p, L_{p}} \beta_{p, 1}^{*} \sigma_{p}^{2} & \beta_{p, L_{p}} \beta_{p, 2}^{*} \sigma_{p}^{2} & \ldots & \left|\beta_{p, L_{p}}\right|^{2} \sigma_{p}^{2}
\end{array}\right] .
\end{aligned}
$$

Because $\operatorname{rank}\left(\mathbf{R}_{p}\right)=1, \operatorname{rank}\left(\mathbf{R}_{S}\right)=P+K_{u}$ and $\operatorname{rank}$ $\left(\mathbf{R}_{X}\right)=P+K_{u}<K$. Thus, the conventional methods for 
DOA estimation fail. Spatial smoothing can let $\operatorname{rank}\left(\mathbf{R}_{X}\right)=$ $K$ to satisfy the requirement of subspace methods, but it requires that the array can be divided into several same subarrays. Thus, in the next section, we will discuss the decomposition of sparse arrays.

\section{Spatial Smoothing in Sparse Arrays}

In this section, we try to divide any sparse array into several subarrays and summarize two situations. The first is that the sparse array is composed of several same sparse subarrays. The second is that the sparse array can be divided into several uniform sparse linear arrays, where although the structure of ULA is different, the process of spatial smoothing is applied to each ULA, and the ULAs with different interelement spacings are applied to remove the ambiguous values.

3.1. Sparse Arrays with Identical Sparse Subarrays. There exists a type of sparse arrays, which are made up of several same arrays. The sparse subarray can be CLAs, NLAs, MRAs, and so on. If the cascade number of array is $Q$, we have $M=Q \bar{M}$ and each stage contains $\bar{M}$ physical sensors, whose location set can be $\mathbb{D}_{q}=\left\{\mathrm{d}_{q \bar{M}}, \mathrm{~d}_{q \bar{M}+1}, \ldots, \mathrm{d}_{q \bar{M}+\bar{M}-1}\right\}$. MSC-
DiSA and GNSA, which are two typical arrays, have different structure rules, but they are the same when the subarray is MRA with four sensors and the displacement is the function to the location of MRA with three sensors. The structures are shown in Figure 1(a). Thus, the received data in (1) can be rewritten as

$$
\mathbf{X}=\left[\begin{array}{c}
\mathbf{X}_{0} \\
\vdots \\
\mathbf{X}_{Q-1}
\end{array}\right]=\left[\begin{array}{c}
\mathbf{A}_{0} \\
\vdots \\
\mathbf{A}_{Q-1}
\end{array}\right] \mathbf{S}+\left[\begin{array}{c}
\mathbf{N}_{0} \\
\vdots \\
\mathbf{N}_{Q-1}
\end{array}\right]
$$

where

$$
\mathbf{A}_{q}=\left[\mathbf{a}_{\mathbb{D}_{q}}\left(\theta_{1,1}\right), \ldots, \mathbf{a}_{\mathbb{D}_{q}}\left(\theta_{P, L_{P}}\right), \mathbf{a}_{\mathbb{D}_{q}}\left(\theta_{K_{c}+1}\right), \ldots, \mathbf{a}_{\mathbb{D}_{q}}\left(\theta_{K}\right)\right]
$$

Each subarray meets the feature of rotational invariance, which can be expressed by

$$
\mathbf{A}_{q}=\mathbf{A}_{0} \Phi_{q}
$$

where

$$
\boldsymbol{\Phi}_{q}=\operatorname{diag}\left[e^{-j \pi \Delta d_{q} \sin \theta_{1,1}}, \ldots, e^{-j \pi \Delta d_{q} \sin \theta_{P, L_{P}}}, e^{-j \pi \Delta d_{q} \sin \theta_{K_{c}+1}}, \ldots, e^{-j \pi \Delta d_{q} \sin \theta_{K}}\right],
$$

where $\Delta d_{q}=d_{q \bar{M}}-d_{0}$ is displacement between the $q$ th subarray and first subarray and $q=0, \ldots, Q-1$. Thus, the spatial smoothing covariance matrix is defined as

$$
\begin{aligned}
\mathbf{R}_{S S} & =\frac{1}{J Q} \sum_{q=0}^{Q-1} \mathbf{X}_{q} \mathbf{X}_{q}^{H}, \\
& =\frac{1}{J Q} \mathbf{A}_{0}\left(\sum_{q=0}^{Q-1} \boldsymbol{\Phi}_{q} \mathbf{S S}^{H} \boldsymbol{\Phi}_{q}^{H}\right) \mathbf{A}_{0}^{H}+\sigma_{n}^{2} \mathbf{I}_{\bar{M}} \\
& =\frac{1}{J Q} \mathbf{A}_{0} \bar{R}_{S} \mathbf{A}_{0}^{H}+\sigma_{n}^{2} \mathbf{I}_{\bar{M}},
\end{aligned}
$$

where $\Phi_{0}=\mathbf{I}_{K}$. In order to let $\operatorname{rank}\left(\mathbf{R}_{S S}\right)=K$, we give the following theorem.

Theorem 1. If $g c d\left(\Delta d_{1}, \ldots, \Delta d_{Q-1}\right)=1, Q \geq \max \left(L_{p}\right)$, and $\bar{M} \geq K, \operatorname{rank}\left(\mathbf{R}_{S S}\right)=K$.

Proof. See Appendix A.

Considering the requirement for setting the displacement between the subarrays [15], we have known that $\operatorname{gcd}\left(\Delta d_{1}, \ldots, \Delta d_{Q-1}\right)=1$. So, when $Q \geq \max \left(L_{p}\right)$ and $\bar{M}>K$, we can estimate all DOAs $\widehat{\theta}_{k}$ by applying subspace methods [32] to $\mathbf{R}_{S S}$.
3.2. Sparse Arrays with ULA-Subarrays. The sparse arrays, which consist of Q ULA-subarrays, are capable to use spatial smoothing algorithm to solve coherent signals. We first give the general model of sensors location denoted as

$$
\mathbb{D}=\bigcup_{q=1}^{Q}\left\{G_{q}+m g_{q} \mid 0 \leq m \leq M_{q}-1\right\}
$$

where $G_{q}$ is the displacement between the $q$ th ULA-subarray and the first ULA-subarray, $M_{q}$ is the sensor number of $q$ th ULA-subarray, and $g_{q}$ is the interelement spacing of $q$ th ULA-subarray.

3.2.1. Sparse Arrays with Two ULA-Subarrays. Figure 1(b) shows four arrays with 12 sensors using two ULAs. Thus, two subarrays, respectively, have $M_{1}$ and $M_{2}$ sensors. The location of subarrays can be denoted as $\mathbb{D}_{1}=\left\{m_{1} g_{1} \mid 0 \leq m_{1} \leq M_{1}-1\right\}, \quad \mathbb{D}_{2}=\left\{G_{2}+m_{2} g_{2} \mid 0 \leq m_{2} \leq\right.$ $\left.M_{2}-1\right\}$, where $g_{1}, g_{2}$ are coprime integers, and generally $G_{1}=0$. The parameters of arrays are defined in Table 2 . Then, the received data in (1) can be rewritten as

$$
\mathbf{X}=\left[\begin{array}{l}
\mathbf{X}_{1} \\
\mathbf{X}_{2}
\end{array}\right]=\left[\begin{array}{l}
\mathbf{A}_{1} \\
\mathbf{A}_{2}
\end{array}\right] \mathbf{S}+\left[\begin{array}{l}
\mathbf{N}_{1} \\
\mathbf{N}_{2}
\end{array}\right],
$$

where 


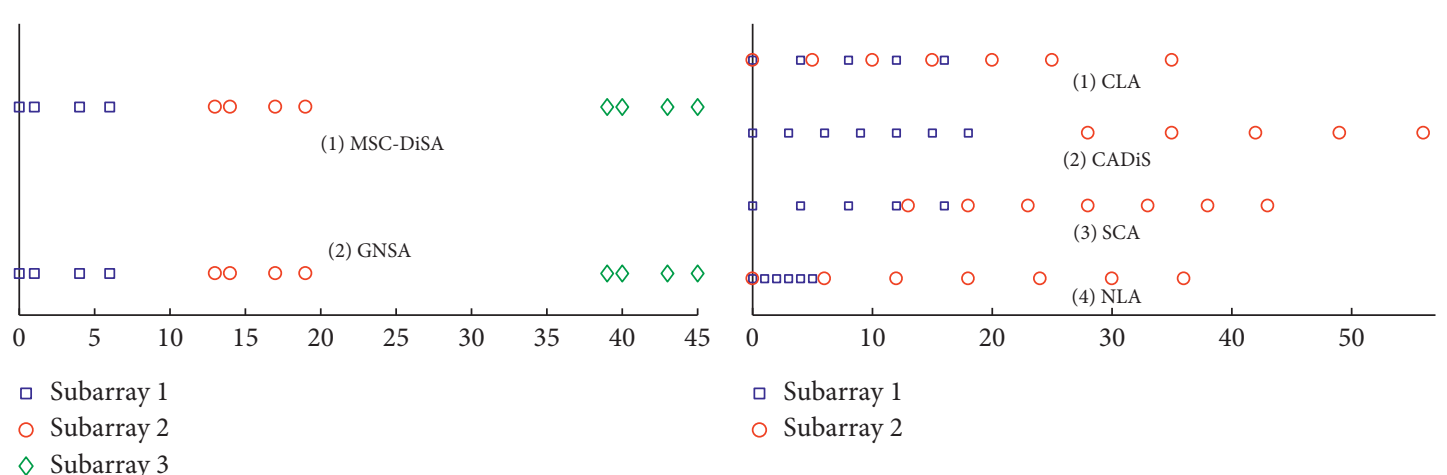

(a)

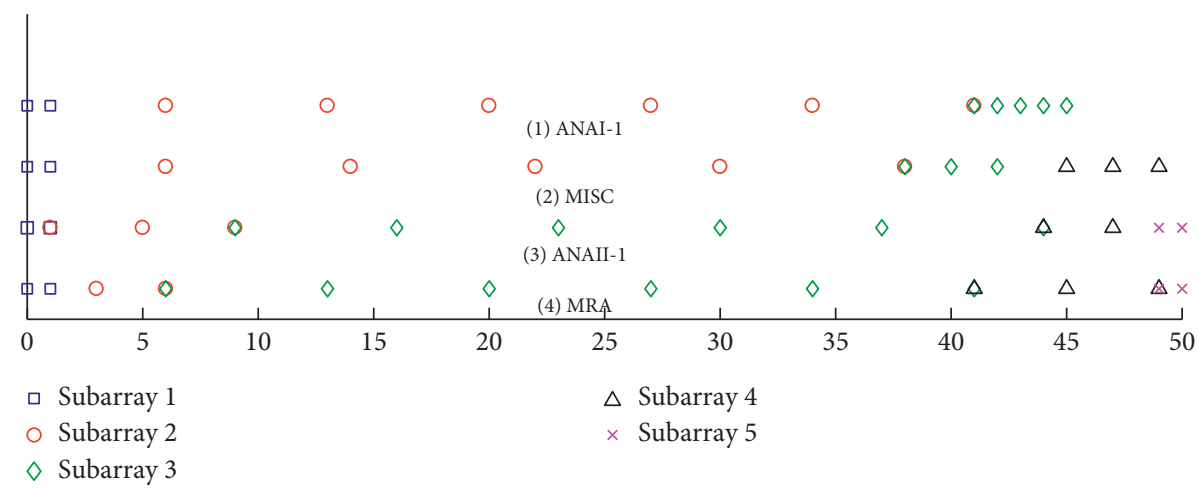

(c)

Figure 1: The structure of sparse arrays when $M=12$.

TABLE 2: Parameters of sparse arrays.

\begin{tabular}{lc}
\hline Type of sparse array & $M_{1}, M_{2}, g_{1}, g_{2}, G_{2}$ \\
\hline CLA & $7,5,4,5,0$ \\
CADis & $5,7,7,3,10$ \\
SCA & $5,7,5,4,8$ \\
NLA & $6,6,1,6,0$ \\
\hline
\end{tabular}

$$
\mathbf{A}_{i}=\left[\mathbf{a}_{\mathbb{D}_{i}}\left(\theta_{1,1}\right), \ldots, \mathbf{a}_{\mathbb{D}_{i}}\left(\theta_{P, L_{P}}\right), \mathbf{a}_{\mathbb{D}_{i}}\left(\theta_{K_{c}+1}\right), \ldots, \mathbf{a}_{\mathbb{D}_{i}}\left(\theta_{K}\right)\right]
$$

Then, the spatial smoothing covariance matrix with size $M_{S_{i}} \times M_{S_{i}}$ of $i$ th subarray is given by

$$
\begin{aligned}
\mathbf{R}_{S S_{i}} & =\frac{1}{J\left(M_{i}-M_{S_{i}}+1\right)} \sum_{m=0}^{M_{i}-M_{S_{i}}}\left(\Gamma_{m}^{i} \mathbf{X}_{i}\right)\left(\Gamma_{m}^{i} \mathbf{X}_{i}\right)^{H} \\
& =\mathbf{A}_{S_{i}} \bar{R}_{S_{i}} \mathbf{A}_{S_{i}}^{H}+\sigma_{n}^{2} \mathbf{I}_{M_{S_{i}}}
\end{aligned}
$$

where $\Gamma_{m}^{i}=\left[0_{M_{S_{i}} \times\left(M_{i}-M_{S_{i}}-m\right)}, \mathbf{I}_{M_{S_{i}}}, 0_{M_{S_{i}} \times m}\right] . \mathbf{A}_{S_{1}}$ is given by

$$
\mathbf{A}_{S_{1}}=\left[\mathbf{a}_{\mathbb{S}_{S_{1}}}\left(\theta_{1,1}\right), \ldots, \mathbf{a}_{\mathbb{D}_{S_{1}}}\left(\theta_{P, L_{P}}\right), \mathbf{a}_{\mathbb{D}_{S_{1}}}\left(\theta_{K_{c}+1}\right), \ldots, \mathbf{a}_{\mathbb{D}_{S_{1}}}\left(\theta_{K}\right)\right],
$$

where $\mathbb{D}_{S_{1}}=\left\{m_{1} g_{1} \mid 0 \leq m_{1} \leq M_{S_{1}}-1\right\}$. Also, we define $\mathbf{A}_{S_{2}}=\bar{A}_{S_{2}} \mathbf{D}$, where

$$
\begin{aligned}
& \bar{A}_{S_{2}}=\left[\mathbf{a}_{\mathbb{D}_{S_{2}}}\left(\theta_{1,1}\right), \ldots, \mathbf{a}_{\mathbb{D}_{S_{2}}}\left(\theta_{P, L_{P}}\right), \mathbf{a}_{\mathbb{D}_{S_{2}}}\left(\theta_{K_{c}+1}\right), \ldots, \mathbf{a}_{\mathbb{D}_{S_{2}}}\left(\theta_{K}\right)\right], \\
& \text { where } \mathbb{D}_{S_{2}}=\left\{m_{2} g_{2} \mid 0 \leq m_{2} \leq M_{S_{2}}-1\right\} \text {, and } \\
& \mathbf{D}=\operatorname{diag}\left[e^{j \pi G_{2} \sin \theta_{1,1}}, \ldots, e^{j \pi G_{2} \sin \theta_{P, L_{P}}}, e^{j \pi G_{2} \sin \theta_{K_{c}+1}}, \ldots, e^{j \pi G_{2} \sin \theta_{K}}\right] .
\end{aligned}
$$

Moreover,

$$
\bar{R}_{S_{i}}=\frac{1}{J\left(M_{i}-M_{S_{i}}+1\right)} \sum_{m=0}^{M_{i}-M_{S_{i}}} \boldsymbol{\Psi}_{i}^{m} \mathbf{S S}^{H}\left(\boldsymbol{\Psi}_{i}^{m}\right)^{H}
$$

where $\quad \Psi_{1}=\operatorname{diag}\left[e^{j \pi g_{1} \sin \theta_{1,1}}, \ldots, e^{j \pi g_{1} \sin \theta_{K}}\right] \quad$ and $\Psi_{2}=\operatorname{diag}\left[e^{j \pi g_{2} \sin \theta_{1,1}}, \ldots, e^{j \pi g_{2} \sin \theta_{K}}\right]$.

Based on the model above, the following statement gives the applicability of using spatial smoothing in this type of sparse array. Firstly, we introduce Theorem 2 in [30] and propose Theorem 3.

Theorem 2. Let $g_{1}$ and $g_{2}$ denote the interelement spacing of two ULAs, respectively. Let $\Theta_{k}^{(1)}=\left\{\theta_{k, 1}^{(1)}, \ldots, \theta_{k, 2 g_{1}-1}^{(1)}\right\}$ and $\Theta_{k}^{(2)}=\left\{\theta_{k, 1}^{(2)}, \ldots, \theta_{k, 2 g_{2}-1}^{(2)}\right\}$ denote the estimated DOA values of the kth signal of two subarrays, respectively, where each set has multiple ambiguous values and one real value. If $\operatorname{gcd}\left(g_{1}, g_{2}\right)=1, \Theta_{k}^{(1)} \cap \Theta_{k}^{(2)}=\theta_{k}$. 
Theorem 3. Let the directions of sources have a random distribution. When $M_{i}-M_{S_{i}}+1 \geq \max \left(L_{p}\right)(p=1, \ldots, P)$ and $M_{S_{i}} \geq K, \operatorname{rank}\left(\bar{R}_{S S_{i}}\right)=K$.

Proof. See Appendix B.

When $\quad M_{i}-M_{S_{i}}+1 \geq \max \left(L_{p}\right)(p=1, \ldots, P) \quad$ and $M_{S_{i}}>K, \operatorname{rank}\left(\bar{R}_{\mathrm{S}_{\mathrm{i}}}\right)=K$ and the requirement of using subspace methods is satisfied. Also, $\operatorname{rank}\left(\mathbf{D} \bar{R}_{S_{2}} \mathbf{D}^{H}\right)=\operatorname{rank}\left(\bar{R}_{S_{2}}\right)$. Then, we know that $g_{1}, g_{2}$ are coprime integers, so we use the subspace method to $\bar{R}_{S S_{i}}$ and obtain the estimated values of the $k$ th signals, defined as $\Theta_{k}^{(1)}=\left\{\theta_{k, 1}^{(1)}, \ldots, \theta_{k, 2 g_{1}-1}^{(1)}\right\}$ and $\Theta_{k}^{(2)}=\left\{\theta_{k, 1}^{(2)}, \ldots, \theta_{k, 2 g_{2}-1}^{(2)}\right\}$. From Theorem 2, common peak finding [30] tells that the real value $\widehat{\theta}_{k}=\Theta_{k}^{(1)} \cap \Theta_{k}^{(2)}$.

3.2.2. Sparse Arrays with Q ULA-Subarrays. When it comes to the sparse arrays with $Q(Q>2)$ ULAs-subarrays, we need to point out that the sensor location of existing sparse arrays satisfies (13). We just give the examples about ANAI-1, MISC, ANAII-1, and MRA with $M=12$ in Table 3.

Based on the analysis in sparse arrays with two subarrays, we just need to select two subarrays, whose interelement spacings are coprime integers, to solve the DOA estimation. Besides the requirement for setting the inter-element spacing, another two criteria for subarray parameters selection are the number of sensors that are as large as possible and the subarray apertures that are as large as possible. The former is to estimate as many sources as possible, and the latter is to ensure the accuracy of estimation. Thus, we can obtain the receiving data $\mathbf{X}_{1}$ and $\mathbf{X}_{2}$ of two chosen subarrays from $\mathbf{X}$. Then, we can use (16) to calculate the spatial smoothing covariance matrix and apply common peak finding [30] to find the DOAs of coherent signals.

\section{Performance Analysis and Simulation Experiments}

4.1. Performance Analysis. We discuss $\max \left(L_{p}\right)$ of each sparse array, that is, the maximum number of detectable coherent signals in one group. For GNSA, $\max \left(L_{p}\right) \leq Q=M / \bar{M}$, so when $Q$ is the maximum integer no more than $\lfloor\sqrt{M}\rfloor, \max \left(L_{p}\right)$ achieving maximum is equal to $Q$. For any sparse array consisting of $Q$ ULAs, we arrange the number of subarray sensors $\left\{M_{1}, \ldots, M_{Q}\right\}$ in descending order and have the set $\left\{\tilde{M}_{1}, \ldots, \tilde{M}_{Q}\right\}$, where $\tilde{M}_{1}>\cdots>\tilde{M}_{Q}$. Based on the theorem that the paper proposes, we can have $\max \left(L_{p}\right) \leq\left\lfloor\tilde{M}_{2} / 2\right\rfloor$ and $K_{c} \leq \tilde{M}_{2}-\left|\tilde{M}_{2} / 2\right|$. Thus, when $P=1$ and $K_{u}=0$, $\max \left(L_{p}\right)=\left\lfloor\tilde{M}_{2} / 2\right\rfloor$. Due to $Q$ subarrays, $\max \left(\tilde{M}_{2}\right)=\lfloor(M-$ $Q+2) / 2\rfloor$ achieves maximum in theory. So, the more the number of ULA-subarrays, the smaller the value of $\max \left(L_{p}\right)$.

Next, we compare the value of $\max \left(L_{p}\right)$ of GNSA with that of CLA, NLA, ANAI-1, MISC, ANAII-1, and MRA. We vary $M$ from 8 to 20 with 2 intervals, and the results are shown in Figure $2 . \max \left(L_{p}\right)$ becomes bigger with the increase of $M$. The CLA and NLA with two ULA-subarrays have the biggest value of $\max \left(L_{p}\right)$. But the ANAII-1 and MRA with 5 or more ULAsubarrays have the smallest value, and only when $M>12$, they can use spatial smoothing to estimate coherent signals. Hence, with a fixed number of sensors, the conclusion that the less subarrays can have the bigger $\max \left(L_{p}\right)$ is corrected.

Moreover, based on the papers, where the sparse arrays are proposed, the maximum array aperture of consecutive virtual ULAs defined as $\Omega$ meets that $\Omega_{\text {MRA }} \geq \Omega_{\text {ANAII-1 }}>\Omega_{\text {MISC }}>\Omega_{\text {ANAI-1 }}>\Omega_{\text {NLA }}>\Omega_{\text {CLA }}$, and $\Omega_{\mathrm{GNSA}}$ is generally between $\Omega_{\mathrm{ANAI}-1}$ and $\Omega_{\mathrm{MISC}}$. When they apply spatial smoothing, the main factor affecting the accuracy is the aperture of smoothing array, defined as $\Pi$. Then, we assume that $P=1$ and $L_{1}=2$ and compare $\Pi$ and $\Pi$ - $\Omega$-ratio $\gamma=\Pi / \Omega$ of different sparse arrays. Figure 3 presents that the more number of ULA-subarrays can achieve bigger $\Omega$, but for spatial smoothing, it is opposite that the less number of ULA-subarrays can achieve bigger $\Pi$.

4.2. Simulation Experiments. We use root mean square error (RMSE) to quantify the accuracy of DOA estimation, given by

$$
\mathrm{RMSE}=\sqrt{\frac{1}{F K} \sum_{f=1}^{F} \sum_{k=1}^{K}\left(\widehat{\theta}_{k, f}-\theta_{k}\right)^{2}},
$$

where $F$ is the Monte Carlo number, $K$ is the number of target signals, and $\widehat{\theta}_{k, f}$ is the DOA of the $k$ th estimated source by the $f$ th Monte Carlo experiment. The simulation conditions are shown in Table 4.

Simulation 1. feasibility of estimate coherent signals in different sparse arrays.

In first simulation, we show the feasibility of estimate coherent signals with maximum number in one group. Thus, assume that $P=1, K_{u}=0$, and $L_{1}$ of each sparse arrays can be seen in Figure 2. Set SNR $=0 \mathrm{~dB}$ and $J=5000$. The estimation values of 100 times experiments are shown in Figure 4 . The figure demonstrates that the sparse arrays can use spatial smoothing to estimate DOAs of coherent signals. Moreover, in the condition of $\max \left(L_{p}\right)$ and low SNR, all estimated values are still close to the real values, which means a favorable performance.

Simulation 2. RMSE performance comparison of different SNRs.

In this simulation, we compare the RMSEs of different sparse arrays and ULAs, when there are both uncorrelated and coherent signals. We set $K=3$, where $\theta_{1,1}=25^{\circ}, \theta_{1,2}=0^{\circ}, \theta_{3}=15^{\circ}$, and $J=5000$. Because ANAII1 and MRA cannot estimate 3 signals after spatial smoothing, we only compare the other arrays, and the results are shown in Figure 5. Obviously, the RMSEs decrease with the increase of SNR. GNSA has the highest RMSE due to the smallest $\Pi$. ULA has the second smallest $\Pi$ as 19, so the RMSE of it is just lower than that of GNSA. The other three sparse arrays have the close RMSE due to their close values of $\Pi$. 
TABle 3: Parameters of sparse arrays.

\begin{tabular}{lcrr}
\hline Arrays & \multicolumn{1}{c}{$\mathbb{D}$} & $Q$ & $\left\{M_{q}, g_{q}, G_{q}\right\}$ \\
\hline \multirow{2}{*}{ ANAI-1 } & $\{0,1\},\{6,13,20,27,34,41\}$ & 3 & $\{2,1,0\},\{7,6,6\},\{5,1,41\}$ \\
& $\{41,42,43,44,45\}$ & & $\{2,1,0\},\{5,8,6\}$ \\
MISC & $\{0,1\},\{6,14,22,30,38\}$ & $\{3,2,38\},\{3,2,45\}$ & $\{1,0\},\{3,4,1\},\{6,7,9\}$, \\
& $\{38,40,42\},\{45,47,49\}$ & & $\{2,2,47\},\{2,1,49\}$ \\
ANAII-1 & $\{0,1\},\{1,5,9\},\{9,16,23,30,37,44\}$ & 5 & $\{2,1,0\},\{2,3,3\},\{6,7,6\}$, \\
& $\{47,49\},\{49,50\}$ & & $\{3,4,41\},\{2,1,49\}$ \\
MRA & $\{0,1\},\{3,6\},\{6,13,20,27,34,41\}$ & 5 & \\
& $\{41,45,49\},\{49,50\}$ & & \\
\hline
\end{tabular}

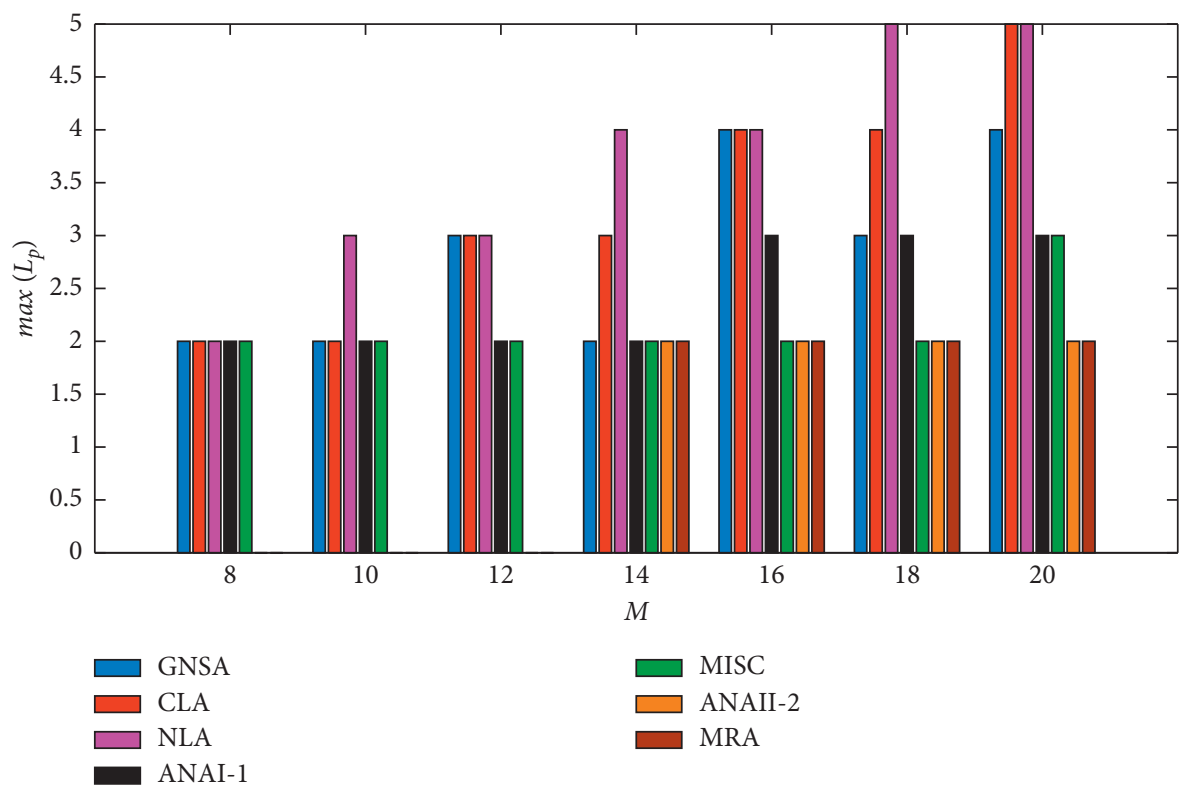

Figure 2: $\max \left(L_{p}\right)$ in different sparse arrays with different $M$.

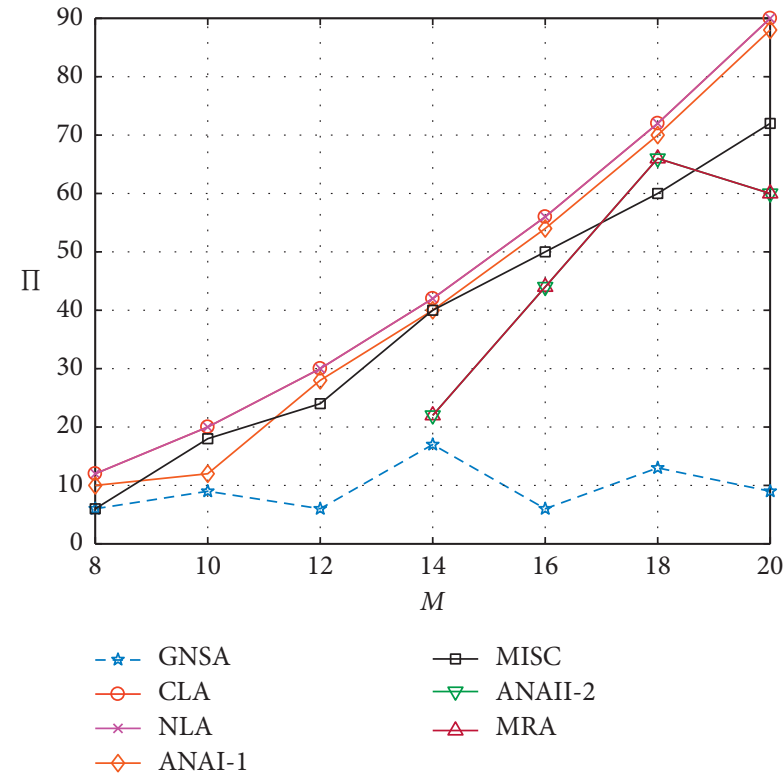

(a)

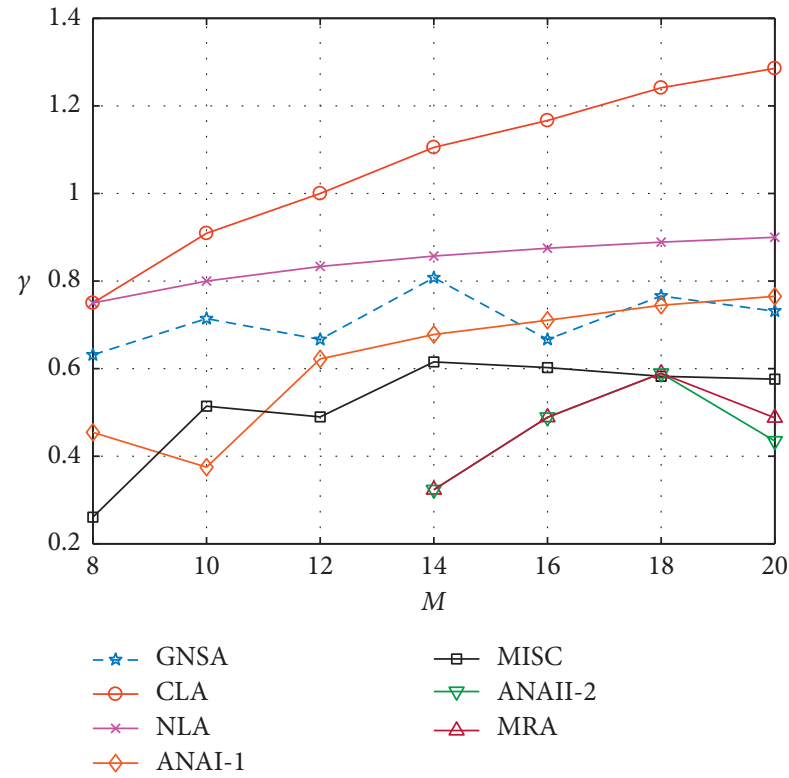

(b)

Figure 3: The comparison of $\Pi$ and $\gamma$ in different sparse arrays. 
TABle 4: Simulation conditions for the experiments.

Simulation parameters

Antenna number

The subarrays for smoothing in ULA

The subarrays for smoothing in GNSA

The subarrays for smoothing in CLA

The subarrays for smoothing in NLA

The subarrays for smoothing in ANAI-1

The subarrays for smoothing in ANAII-1

The subarrays for smoothing in MRA

Monte Carlo number

SNR

Snapshot

Values

$\{0,1,2,3, \ldots, 18,19\}$

$\{0,1,4,7,9\},\{19,20,23,26,28\}$

$\{76,77,80,83,85\},\{114,115,118,121,123\}$

$\{0,10,20,30,40,50,60,70,80,90,100\}$

$\{0,11,22,33,44,55,66,77,88,99\}$

$\{0,1,2,3,4,5,6,7,8,9,10\}$

$\{0,10,20,30,40,50,60,70,80,90,100\}$ '

$\{11,22,33,44,55,66,77,88,99,110\}$

$\{110,111,112,113,114,115,116\}$

$\{35,50,65,80,95,110\}$

$\{3,11,19,27,35\}$

$\{28,43,58,73,88,103\}$

$\{103,111,119,127,135\}$ '

$$
F=100
$$

$\{-5,0,5,10,15\}$

$\{50,100,200,500,1000,2000,5000\}$

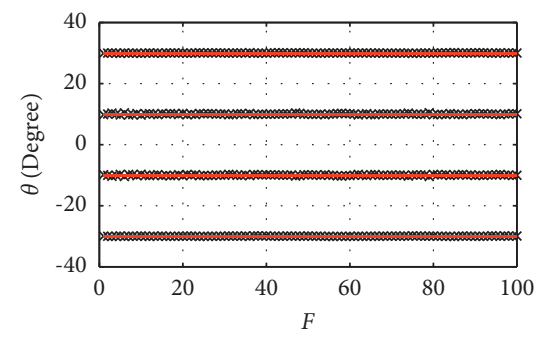

- - - Real Values

$\times \quad$ Estimated Values

(a)

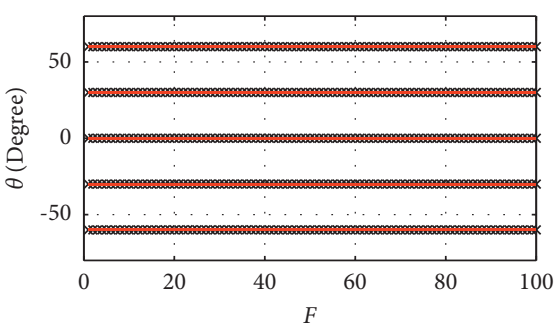

- - Real Values

$\times \quad$ Estimated Values

(c)

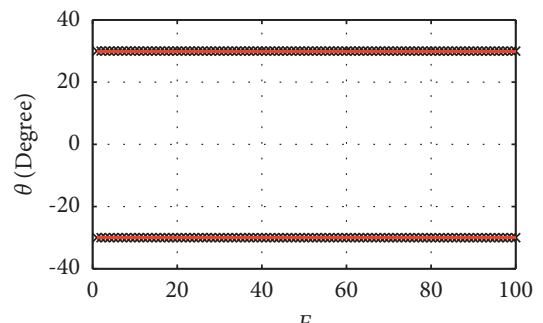

- _ - Real Values

$\times \quad$ Estimated Values

(e)

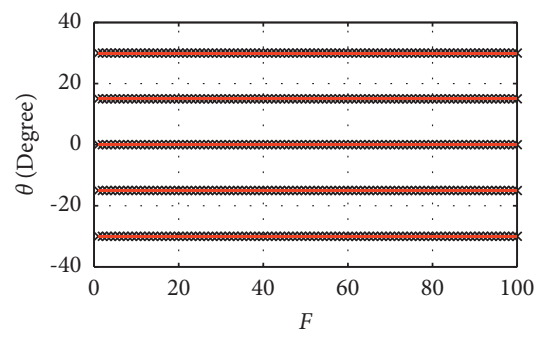

- - Real Values

$\times \quad$ Estimated Values

(b)

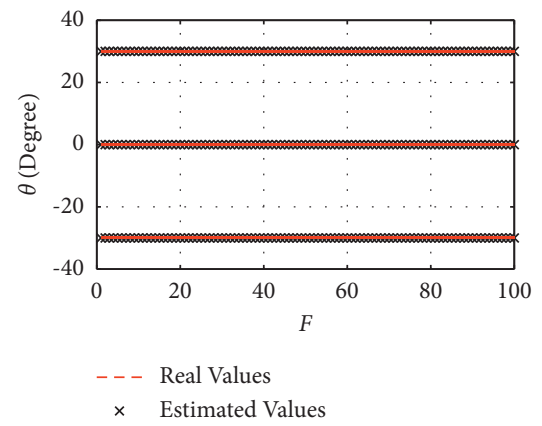

(d)

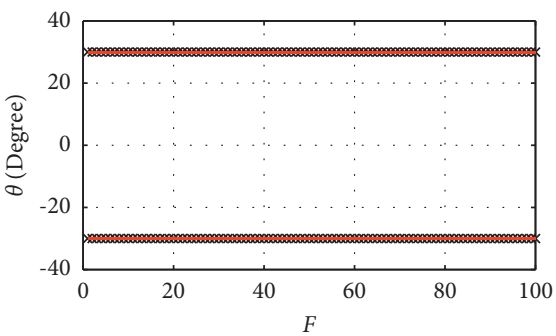

- - - Real Values

$\times \quad$ Estimated Values

(f)

Figure 4: The estimation values of coherent signals in different sparse arrays. 


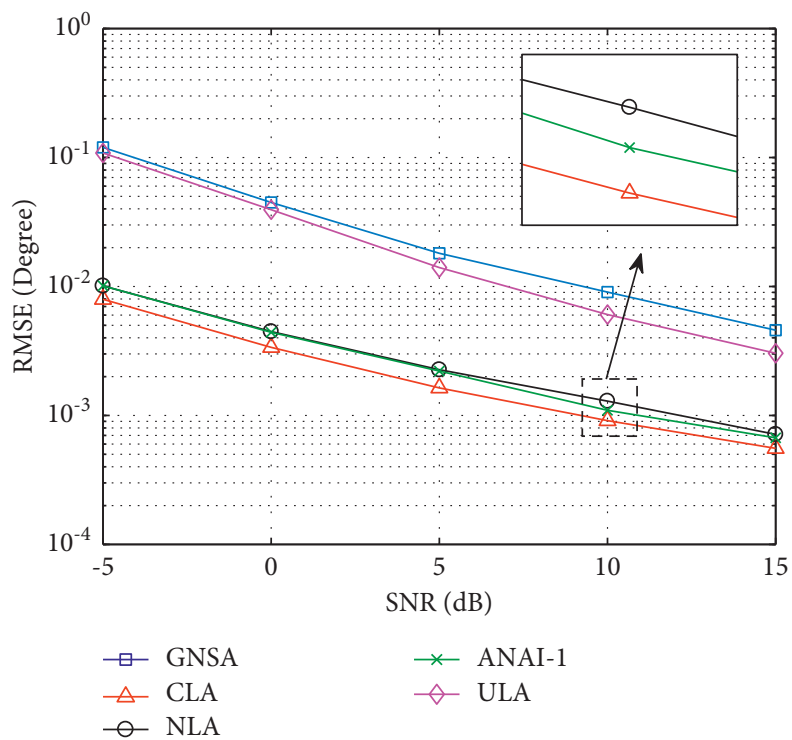

FIGURE 5: The RMSE versus SNR in different sparse arrays using spatial smoothing.

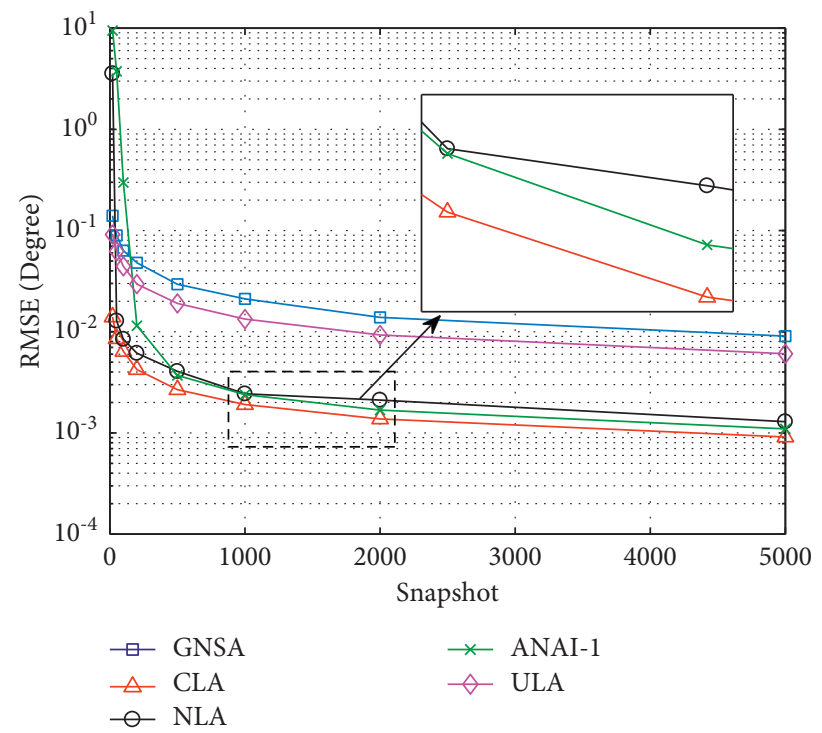

FIgURE 6: The RMSE versus the number of snapshots in different sparse arrays using spatial smoothing.

Simulation 3. RMSE performance comparison of different number of snapshots.

Similar to the simulation 2, we present the RMSEs of different sparse arrays under different snapshots with $\mathrm{SNR}=10 \mathrm{~dB}$. From Figure 6, the RMSEs decrease with the increase of snapshot numbers, but when $J>500$, the downtrend of RMSEs become slow. Other conclusions are the same as those in simulation 2 .

Simulation 4. RMSE performance comparison using FBSS.
In this simulation, we use FBSS to replace spatial smoothing and do simulations 2 and 3 again. FBSS can be seen as an improvement method of spatial smoothing, where $\Pi$ can be 1.5 times that of spatial smoothing [26]. Thus, we do not need to present the analysis in Part 1 of this section about using FBSS. But we should note that FBSS is not applicable to GNSA because it needs that the subarrays have uniform structure, where we still use spatial smoothing in GNSA. The results are shown in Figures 7 and 8. Compared with the results in simulations 2 and 3, FBSS has improved 


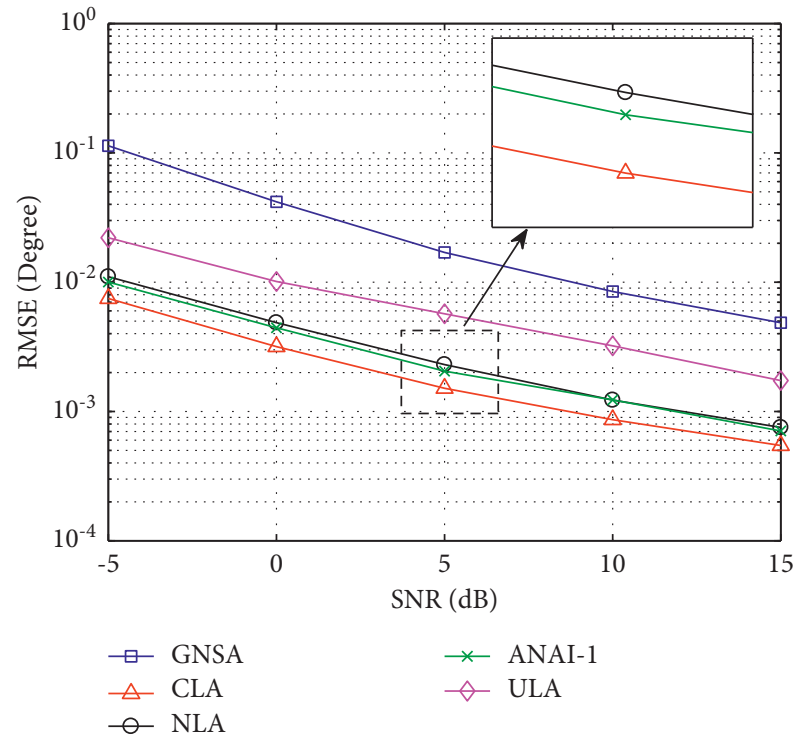

FIGURE 7: The RMSE versus SNR in different sparse arrays using FBSS.

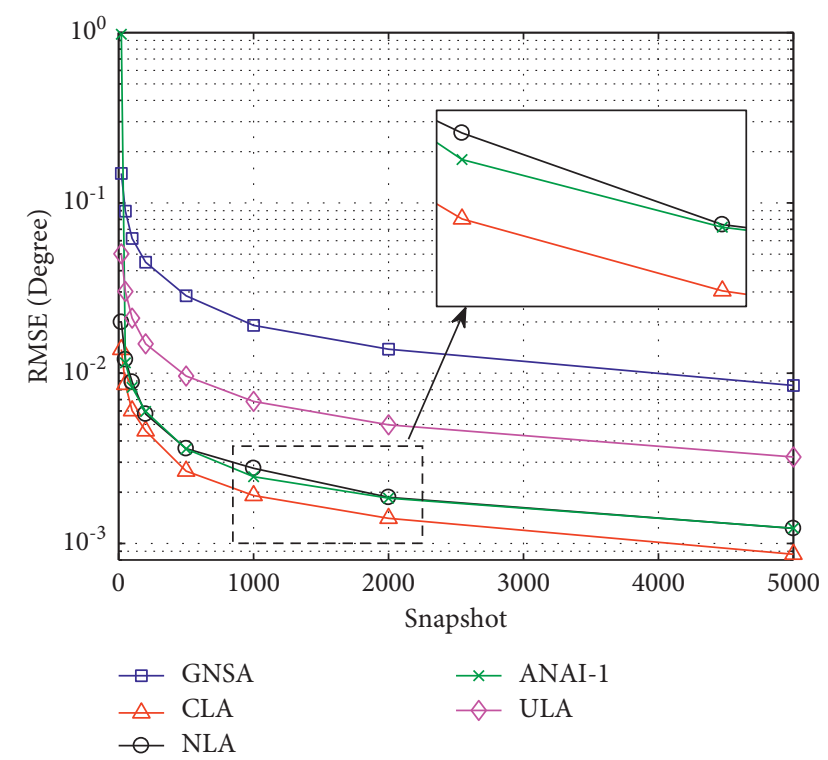

Figure 8: The RMSE versus the number of snapshots in different sparse arrays using FBSS.

the accuracy. Thus, we can use FBSS in sparse arrays to find more coherent signals and obtain higher accuracy.

\section{Conclusions}

In this paper, the DOA estimation methods using spatial smoothing for coherent signals in sparse arrays are proposed. We divide the sparse arrays into two parts. The first type consists of several identical sparse arrays. The second type can be decomposed of several ULA-subarrays. In view of subarrays, spatial smoothing can be applied in sparse arrays. Based on the analysis of the maximum number of detectable coherent signals in one group, the sparse arrays with less subarrays are capable to estimate more signals and own bigger smoothing array aperture. Also, the simulation experiments prove that CLA and NLA have better performance than other arrays.

\section{Appendix}

\section{$\mathbf{A}$}

Proof of Theorem 1. The matrix $\bar{R}_{S}$ can be rewritten as

$$
\bar{R}_{S}=\left[\begin{array}{cccc}
\bar{R}_{1} & & & \\
& \bar{R}_{2} & & \\
& & \ddots & \\
& & & \sigma_{K}^{2}
\end{array}\right],
$$

where $\bar{R}_{S}$ is a block-diagonal matrix and

$$
\bar{R}_{p}=\frac{1}{Q} \sum_{q=0}^{Q-1} \Phi_{q} \mathbf{R}_{p}\left(\Phi_{q}\right)^{H}
$$

Define $\Phi=\operatorname{diag}\left[e^{-j \pi \sin \theta_{1,1}}, \ldots, e^{-j \pi \sin \theta_{K}}\right]$, and (20) can be denoted as

$$
\bar{R}_{p}=\frac{1}{Q} \sum_{q=0}^{Q-1} \Phi^{\Delta d_{q}} \mathbf{R}_{p}\left(\Phi^{\Delta d_{q}}\right)^{H} .
$$

If $\operatorname{rank}\left(\bar{R}_{p}\right)=L_{p}, \operatorname{rank}\left(\bar{R}_{S}\right)=K$. Thus, we rewrite $\bar{R}_{p}$ as

$$
\bar{R}_{p}=\left[\begin{array}{llll}
\mathbf{I}_{K} & \boldsymbol{\Phi}^{\Delta d_{1}} & \ldots & \boldsymbol{\Phi}^{\Delta d_{\mathrm{Q}-1}}
\end{array}\right]\left[\begin{array}{llll}
\mathbf{R}_{p} & & \\
& \ddots & \\
& & \mathbf{R}_{p}
\end{array}\right]\left[\begin{array}{c}
\mathbf{I}_{K} \\
\boldsymbol{\Phi}^{\Delta d_{1}} \\
\vdots \\
\boldsymbol{\Phi}^{\Delta d_{Q-1}}
\end{array}\right]^{H} .
$$

Based on (25), it can also be simplified to give $\bar{R}_{p}=\mathbf{V}_{p} \mathbf{V}_{p}^{H}$, where

$$
\mathbf{V}_{p}=\mathbf{C}_{p} \Phi^{\Delta d_{1}} \mathbf{C}_{p}, \ldots, \Phi^{\Delta d_{Q-1}} \mathbf{C}_{p},
$$

with $\mathbf{C}_{p}$ denoting the Hermitian square root of $\bar{R}_{p}$.

Because $\operatorname{rank}\left(\bar{R}_{p}\right)=\operatorname{rank}\left(\mathbf{V}_{p}\right)$, we need to prove that $\operatorname{rank}\left(\mathbf{V}_{p}\right)=L_{p}$. We take column permutations to $\mathbf{V}_{p}$, which cannot change the rank of a matrix, and have

$$
\operatorname{rank}\left(\mathbf{V}_{\mathbf{p}}\right)=\operatorname{rank}\left(\begin{array}{cccc}
c_{11} \boldsymbol{v}_{1} & c_{12} \boldsymbol{v}_{1} & \ldots & c_{1 L_{p}} \boldsymbol{v}_{1} \\
\vdots & \vdots & \ldots & \vdots \\
c_{L_{p}} \mathbf{v}_{L_{p}} & c_{L_{p} 2} \boldsymbol{v}_{L_{p}} & \ldots & c_{L_{p} L_{p}} \boldsymbol{v}_{L_{p}}
\end{array}\right) \text {, }
$$

where $c_{i, j}$ represents element in the $i$ th row and $j$ th column of $\mathbf{C}_{p}$ and $v$ is expressed as

$$
\boldsymbol{v}_{k}=\left[1, e^{j \pi \Delta d_{1} \sin \theta_{k}}, \ldots, e^{j \pi \Delta d_{\mathrm{Q}-1} \sin \theta_{k}}\right] .
$$

When each row of $\mathbf{C}_{p}$ has at least one nonzero element and vectors $\left\{v_{1}, \ldots, v_{L_{p}}\right\}$ are linearly independent, $\operatorname{rank}\left(\mathbf{V}_{p}\right)=L_{p}$. Because every signal has the positive energy, 
every row of $\mathbf{C}$ cannot be all zeros. The matrix $\left[\nu_{1}, \cdots, v_{L_{p}}\right]$ can be seen as the manifold matrix of a sparse array and $\left\{0, \Delta d_{1}, \ldots, \Delta d_{Q-1}\right\}$ are denoted as the location of sensors.

Hence, we introduce the theorem in $[32,33]$, which tells that when $\operatorname{gcd}\left(\Delta d_{1}, \Delta d_{2}, \ldots, \Delta d_{Q-1}\right)=1$, the array manifold $v_{k}$ is invertible. Invertibility means that if $\theta_{1} \neq \theta_{2}$, then $v_{1} \neq v_{2}$. Then, we can obtain the conclusion that if $Q \geq L_{p}$, the rank of $\left[v_{1}, \ldots, v_{L_{p}}\right]$ is $L_{p}$. So, we prove that if $\operatorname{gcd}\left(\Delta d_{1}, \Delta d_{2}, \ldots, \Delta d_{Q-1}^{p}\right)=1 \quad$ and $\quad Q \geq \max \left(L_{p}\right)$, $\operatorname{rank}\left(\underline{\mathbf{V}}_{p}\right)=L_{p}$, and $\operatorname{rank}\left(\bar{R}_{p}\right)=L_{p}$ for any $p$, then $\operatorname{rank}\left(\bar{R}_{S}\right)=K$. Hence, when $\bar{M} \geq K, \operatorname{rank}\left(\mathbf{R}_{S S}\right)=K$.

\section{B}

Proof of Theorem 3. In this situation, $\bar{R}_{S_{i}}$ is also a blockdiagonal matrix, given by

$$
\bar{R}_{S_{i}}=\left[\begin{array}{cccc}
\bar{R}_{1, i} & & & \\
& \bar{R}_{2, i} & & \\
& & \ddots & \\
& & & \sigma_{K}^{2}
\end{array}\right],
$$

and $\bar{R}_{p, i}$ is changed to

$$
\bar{R}_{p, i}=\frac{1}{M_{i}-M_{S_{i}}+1} \sum_{s=0}^{M_{i}-M_{S_{i}}} \Psi_{\mathbf{i}}^{g_{i} s} \mathbf{R}_{p}\left(\Psi_{\mathbf{i}}^{g_{i} s}\right)^{H} .
$$

Similar to the Proof of Theorem $1, \bar{R}_{p, i}=\mathbf{V}_{p, i} \mathbf{V}_{p, i}^{H}$, where

$$
\mathbf{V}_{p, i}=\left[\mathbf{C}_{p, i} \Psi_{i}^{g_{i}} \mathbf{C}_{p, i}, \ldots, \boldsymbol{\Psi}_{i}^{g_{i}\left(M_{i}-M_{S_{i}}\right)} \mathbf{C}_{p, i}\right] \text {, }
$$

with $\mathbf{C}_{p, i}$ denoting the Hermitian square root of $\mathbf{R}_{p, i}$. We just need to prove that $\operatorname{rank}\left(\mathbf{V}_{p, i}\right)=L_{p}$ because $\operatorname{rank}\left(\bar{R}_{p, i}\right)=\operatorname{rank}\left(\mathbf{V}_{p, i}\right)$. Considering the analysis in Appendix $A$, we should prove that each row of $\mathbf{C}_{p, i}$ has at least one nonzero element and vectors $\left\{v_{1, i}, \ldots, v_{L_{p}, i}\right\}$ are linearly independent, where

$$
\boldsymbol{v}_{k, i}=\left[1, e^{j \pi g_{i} \sin \theta_{k}}, \ldots, e^{j \pi g_{i} \sin \theta_{k}}\right] .
$$

It is easy to obtain that each row of $\mathbf{C}_{p, i}$ has at least one nonzero element. $\left[v_{1, i}, \ldots, v_{L_{p}, i}\right]$ is a Vandermonde matrix and $\left\{0, g_{i}, \ldots, g_{i}\left(M_{i}-M_{S_{i}}\right)\right\}$ are denoted as the location of sensors. Because $\operatorname{gcd}\left(g_{i}, \ldots, g_{i}\left(M_{i}-M_{S_{i}}\right)\right)=g_{i} \geq 1, v_{k}$ is not invertible. For example, if $g_{i}=2, \theta_{1,1}=30^{\circ}$, and $\theta_{1,2}=-30^{\circ}$, then $v_{1, i}=v_{2, i}$ and $\operatorname{rank}\left(\widehat{R}_{p, i}\right)=1$, while if $\theta_{1,2}=-30.1^{\circ}, \operatorname{rank}\left(\widehat{R}_{p, i}\right)=2$. In general, assuming that $g_{i}=\alpha$, if

$$
\sin \theta_{k}-\sin \theta_{l} \neq \frac{2 \pi n}{\alpha}
$$

for any integer $n, v_{k} \neq v_{l}$ and $\operatorname{rank}\left(\widehat{R}_{p, i}\right)=L_{p}$. Considering that the directions of sources generally have a random distribution in real environment, the parameters $\left(\sin \theta_{k}, \sin \theta_{l}\right)$ satisfy (33) with probability one. In other words, if $g_{i}>1$ and $M_{i}-M_{S_{i}}+1 \geq \max \left(L_{p}\right), \operatorname{rank}\left(\bar{R}_{p, i}\right)=$ $L_{p}$ and $\operatorname{rank}\left(\bar{R}_{S_{i}}\right)=K$ in this situation. Hence, when $M_{S_{i}} \geq K, \operatorname{rank}\left(\bar{R}_{S S_{i}}\right)=K$.

\section{Data Availability}

The data used in this article are provided by our simulations and the data used to support the findings of this study are available from the corresponding author upon request.

\section{Conflicts of Interest}

The authors declare that they have no conflicts of interest.

\section{Acknowledgments}

This study was supported by the National Natural Science Foundation of China (grant no. 61401513).

\section{References}

[1] F. Sun, Q. Wu, P. Lan, G. Ding, and L. Chen, "Real-valued doa estimation with unknown number of sources via reweighted nuclear norm minimization," Signal Processing, vol. 148, pp. 48-55, 2018.

[2] F. Sun, P. Lan, and G. Zhang, "Reduced dimension based twodimensional DOA estimation with full DOFs for generalized co-prime planar arrays," Sensors, vol. 18, no. 6, 2018.

[3] A. Moffet, "Minimum-redundancy linear arrays," IEEE Transactions on Antennas and Propagation, vol. 16, no. 2, pp. 172-175, 1968.

[4] P. Vaidyanatha, "Sparse sensing with co-prime samplers and arrays," IEEE Transactions on Signal Processing, vol. 59, no. 2, 2011.

[5] P. Pal and P. Vaidyanatha, "Nested arrays: a novel approach to array processing with enhanced degrees of freedom," IEEE Transactions on Signal Processing, vol. 58, no. 8, pp. 41674181, 2010.

[6] P. Pal and P. Vaidyanatha, "Coprime sampling and the music algorithm," in Proceedings of the Digital Signal Processing Workshop \& IEEE Signal Processing Education Workshop, Rio de Janeiro, Brazil, July 2011.

[7] C. Liu and P. Vaidyanathan, "Remarks on the spatial smoothing step in coarray music," IEEE Signal Processing Letters, vol. 22, no. 9, pp. 1438-1442, 2015.

[8] P. Pal and P. Vaidyanathan, "On application of lasso for sparse support recovery with imperfect correlation awareness," in Proceedings of the 2012 Conference Record of the Forty Sixth Asilomar Conference on Signals, Systems and Computers (ASILOMAR), Pacific Grove, CA, USA, November 2013.

[9] Z. Tan and A. Nehorai, "Sparse direction of arrival estimation using co-prime arrays with off-grid targets," IEEE Signal Processing Letters, vol. 21, no. 1, pp. 26-29, 2013.

[10] J. Yang, G. Liao, and J. Li, "An efficient off-grid doa estimation approach for nested array signal processingby using sparse bayesian learning strategies," Signal Processing, vol. 128, pp. 110-122, 2016.

[11] Y. Zhang, S. Qin, and M. Amin, "Doa estimation exploiting coprime arrays with sparse sensor spacing," in Proceedings of the IEEE International Conference on Acoustics, Florence, Italy, May 2014. 
[12] S. Qin, Y. Zhang, and M. Amin, "Generalized coprime array configurations for direction-of-arrival estimation," IEEE Transactions on Signal Processing, vol. 63, no. 6, 2015.

[13] S. Ren, W. Wang, and Z. Chen, "Doa Estimation Exploiting Unified Coprime Array with Multi-Period Subarrays," in Proceedings of the 2016 CIE International Conference on Radar (RADAR), Guangzhou, China, October 2016.

[14] S. Alamoudi, A. Aldhaheri, S. Alawsh, and A. Muqaibel, "Sparse doa estimation based on ashifted coprime array configuration," in Proceedings of the Microwave Symposium, Honolulu, HI, USA, June 2017.

[15] Junpeng, G. Hu, X. Zhang, and H. Zhou, "Generalized nested array: optimization for degrees of freedom and mutual coupling," IEEE Communications Letters, vol. 22, no. 6, pp. 1208-1211, 2018.

[16] H. Xu, W. Cui, F. Mei, B. Ba, and C. Jian, "The design of A novel sparse array using two uniform linear arrays considering mutual coupling," Journal of Sensors, vol. 2021, Article ID 9934097, 15 pages, 2021.

[17] C. Liu and P. Vaidyanathan, "Super nested arrays: linear sparse arrays with reduced mutual coupling part i:Fundamentals," IEEE Transactions on Signal Processing, vol. 64, no. 15, pp. 3997-4012, 2016.

[18] C. Liu and P. Vaidyanathan, "Super nested arrays: linear sparse arrays with reduced mutual coupling part ii: high-order extensions," IEEE Transactions on Signal Processing, vol. 64, no. 15, 2016.

[19] J. Liu, Y. Zhang, Y. Lu, S. Ren, and S. Cao, "Augmented nested arrays with enhanced dof and reduced mutual coupling," IEEE Transactions on Signal Processing, vol. 65, no. 21, pp. 5549-5563, 2017.

[20] Z. Zheng, W. Wang, Y. Kong, and Y. Zhang, "Misc array: a new sparse array design achieving increased degrees of freedom and reduced mutual coupling effect," IEEE Transactions on Signal Processing, vol. 67, no. 7, pp. 1728-1741, 2019.

[21] M. Yang, A. Haimovich, B. Chen, and X. Yuan, "A new array geometry for DOA estimation with enhanced degrees of freedom," in Proceedings of the 2016 IEEE International Conference on Acoustics Speech and Signal Processing (ICASSP), pp. 3041-3045, Shanghai, China, March 2016.

[22] M. Yang, J. Ding, B. Chen, and X. Yuan, "A multiscale sparse array of spatially spread electromagnetic-vector-sensors for direction finding and polarization estimation," IEEE Access, vol. 6, pp. 9807-9818, 2018.

[23] M. Yang, A. M. Haimovich, X. Yuan, L. Sun, and B. Chen, “A unified array geometry composed of multiple identical subarrays with hole-free difference coarrays for underdetermined DOA estimation," IEEE Access, vol. 6, pp. 14238-14254, 2018.

[24] Y. Zhang, H. Xu, R. Zong, B. Ba, and D. Wang, "A novel high degree of freedom sparse array with displaced multistage cascade subarrays," Digital Signal Processing, vol. 90, pp. 36-45, 2019.

[25] T. SHAN, "On spatial smoothing for direction-of-arrival estimation of coherent signals," IEEE Trans.acoust.speech Signal Processing, vol. 33, no. No. 4, pp. 806-811, 1985.

[26] U. Pillai and H. Kwon, "Forward/backward spatial smoothing techniques for coherent signal identification," IEEE Transactions on Acoustics, Speech, \& Signal Processing, vol. 37, no. 1, pp. 8-15, 1989.

[27] F. Fang-Ming Han and X. Xian-Da Zhang, "An esprit-like algorithm for coherent doa estimation," IEEE Antennas and Wireless Propagation Letters, vol. 4, pp. 443-446, 2005.
[28] H. Xu, D. Wang, B. Ba, W. Cui, and Y. Zhang, "Direction-ofarrival estimation for both uncorrelated and coherent signals in coprime array," IEEE Access, vol. 7, pp. 18590-18600, 2019.

[29] Y. Hu, Y. Liu, and X. Wang, "DOA estimation of coherent signals on coprime arrays exploiting fourth-order cumulants," Sensors, vol. 17, no. 4, 2017.

[30] C. Zhou, Z. Shi, Y. Gu, and X. Shen, "Decom: doa estimation with combined music for coprime array," in Proceedings of the International Conference on Wireless Communications \& Signal Processing, Hangzhou, China, July 2013.

[31] Z. Zheng, Y. Huang, W.-Q. Wang, and H. C. So, "Directionof-arrival estimation of coherent signals via coprime array interpolation," IEEE Signal Processing Letters, vol. 27, pp. 585-589, 2020.

[32] P. Vaidyanathan and P. Pal, "Direct-music on sparse arrays," in Proceedings of the International Conference on Signal Processing \& Communications, Bangalore, India, October 2012.

[33] P. Vaidyanathan and P. Pal, "Why does direct-music on sparse-arrays work?" in Proceedings of the Conference on Signals, Pacific Grove, CA, USA, November 2013. 